Article

\title{
Ethyl Acetate Fraction of Amomum xanthioides Ameliorates Nonalcoholic Fatty Liver Disease in a High-Fat Diet Mouse Model
}

\author{
Hwi-Jin Im ${ }^{\dagger}$, Seung-Ju Hwang ${ }^{+}{ }^{\circ}$, Jin-Seok Lee, Sung-Bae Lee, Ji-Yun Kang \\ and Chang-Gue Son * \\ Liver and Immunology Research Center, Dunsan Oriental Hospital of Daejeon University Daedukdae-ro 176 \\ bun-gil 75, Seo-gu, Daejeon 35353, Korea; lastdohee@gmail.com (H.-J.I.); bluesea9292@naver.com (S.-J.H.); \\ neptune@dju.kr (J.-S.L.); sky161300@naver.com (S.-B.L.); kangjy0118@naver.com (J.-Y.K.) \\ * Correspondence: ckson@dju.kr; Tel.: +82-42-257-6397; Fax: +82-42-257-6398 \\ + These authors contributed equally to this study.
}

Received: 21 July 2020; Accepted: 11 August 2020; Published: 13 August 2020

check for updates

\begin{abstract}
The global prevalence of nonalcoholic fatty liver disease (NAFLD) is estimated to be $25 \%$ and has continued to increase; however, no drugs have yet been approved for NAFLD treatments. The ethyl acetate fraction of Amomum xanthioides (EFAX) was previously reported to have an anti-hepatic fibrosis effect, but its effects on steatosis or steatohepatitis remain unclear. This study investigated the anti-fatty liver of EFAX using a high-fat diet mouse model. High-fat diet intake for 8 weeks induced hepatic steatosis with mild inflammation and oxidative damage and increased the adipose tissue weight along with the development of dyslipidemia. EFAX treatment significantly ameliorated the steatohepatic changes, the increased weight of adipose tissues, and the altered serum lipid profiles. These observed effects were possibly due to the lipolysis-dominant activity of EFAX on multiple hepatic proteins including sterol regulatory element-binding protein (mSREBP)-1c, peroxisome proliferator-activated receptor (PPAR)- $\alpha$, AMP-activated protein kinase, and diglyceride acyltransferases (DGATs). Taken together, these results show that EFAX might be a potential therapeutic agent for regulating a wide spectrum of NAFLDs from steatosis to fibrosis via multiple actions on lipid metabolism-related proteins. Further studies investigating clear mechanisms and their active compounds are needed.
\end{abstract}

Keywords: NAFLD; NASH; fatty liver; herb; Amomum xanthioides

\section{Introduction}

Nonalcoholic fatty liver disease (NAFLD) is becoming a major health problem because of its high estimated global prevalence of $25 \%$ and continuous increasing pattern over the last decade [1]. Recently, the economic burden of NAFLD has been reported to be over 100 billion dollars in the USA [2]. Furthermore, NAFLD is crucially linked to the development of not only chronic hepatic diseases but also obesity, type 2 diabetes, cardiovascular diseases, and chronic kidney diseases [3-5].

Weight loss through lifestyle modification (dietary habits and physical exercise) is the first-line treatment for NAFLD [6]. To improve fibrosis and steatosis, weight loss of $10 \%$ or more is required [7]. However, a previous study reported that only $10 \%$ of patients achieved a weight reduction of more than $10 \%$, and not all patients gained beneficial effects from the weight reduction [8]. In addition, 8 19\% of nonobese subjects, especially in Asia, are also diagnosed with NAFLD [9]. Therefore, although weight loss through lifestyle modification is a very effective therapy for NAFLD, pharmacological intervention in addition to lifestyle modification is also recommended for patients with NAFLD [10]. 
Clinically, vitamin E and pioglitazone have been recommended for patients with NAFLD by American and European guidelines [11,12]. The above two drugs were reported to show beneficial effects on nonalcoholic fatty liver (NAFL) and nonalcoholic steatohepatitis (NASH). However, there is insufficient evidence on their modulation of fibrosis or cirrhosis [13], and several adverse effects including increased mortality or cancer risk have been reported [14-16]. Therefore, the development of other treatments is still required.

A total of 395 clinical trials were registered at ClinicalTrials.gov (at 10 June 2020) for the investigation of a potential drug for NAFLD. However, to date, no agent has been approved for the treatment of NAFLD. A major challenge for NAFLD drug development is the complexity and multitude of therapeutic targets; therefore, combination therapy or herbal medicine is considered a promising therapy for NAFLD [17-19].

Amomum xanthioides is a medicinal herb that has been reported to show gastrointestinal protection, liver protective, and anti-dyslipidemic effects [20]. Moreover, our previous studies proved that one A. xanthioides fraction has anti-hepatofibrotic activity in dimethylnitrosamine (DMN) and bile duct-ligation animal models [21,22]. Hepatic fibrosis is considered an advanced pathologic stage of NAFLD [13]. We thus hypothesized that $A$. xanthioides is able to control NAFL and NASH.

We herein investigated the anti-NAFLD effects of $A$. xanthioides using a high-fat diet mouse model.

\section{Materials and Methods}

\subsection{Preparation and Fingerprinting of A. xanthioides}

A. xanthioides was purchased from Jeong-Seong Pharmaceutical Company (Daejeon, Korea). After screening the optimized fractions, the ethyl acetate fraction of A. xanthioides (EFAX) was selected and extracted using an organic solvent extraction method (Supplementary Figure S1). Briefly, $10 \mathrm{~kg}$ of ground A. xanthioides was extracted in $100 \mathrm{~L}$ of absolute methanol for 1 week. One hundred milliliters of distilled water (DW) was mixed with $900 \mathrm{~mL}$ of the methanol fraction. Then, $100 \mathrm{~mL}$ of a petroleum ether extract was mixed with $900 \mathrm{~mL}$ of DW $(1 \mathrm{~L})$ and further fractionated with ethyl acetate $(1 \mathrm{~L})$ to isolate EFAX. EFAX was stored at $-70^{\circ} \mathrm{C}$ in the Liver \& Immunology Research Center, Daejeon Oriental Hospital of Daejeon University (Storage specimen \#EFAX 2017-03, Liver and Immunology Research Center, Daejeon, Korea). The final fraction yields were $0.19 \%(w / w)$.

To confirm the reproducibility of the EFAX chemical composition, ultrahigh-performance liquid chromatography-tandem mass spectrometry (UHPLC-MS/MS, Thermo Scientific, Waltham, CA, USA) was performed as described previously [21]. Briefly, quantitative analysis of the major three compounds in EFAX was performed using UHPLC-MS/MS. The chromatogram indicated that the main chemical components of EFAX were procyanidin B2, catechin, and quercitrin and occurred at 4.31, 4.75, and $7.24 \mathrm{~min}$, respectively. The molecular weights of the three chemicals were confirmed as follows: procyanidin B2, $578.17 \mathrm{~g} / \mathrm{mol}$; catechin, $290.08 \mathrm{~g} / \mathrm{mol}$; and quercitrin, $448.11 \mathrm{~g} / \mathrm{mol}$ (Supplementary Figure S2).

\subsection{Chemicals and Reagents}

The reagents for the present study were as follows: TRI reagent (Invitrogen, Carlsbad, CA, USA); RIPA buffer (LPS solution, Korea); bovine serum albumin (BSA, GenDEPOT, Katy, TX, USA); n-butanol (J.T. Baker, Phillpsburg, NJ, USA); Mayer's hematoxylin, methanol, and isopropanol (Wako Pure Chemical Industries, Osaka, Japan); and phosphoric acid and potassium chloride (SAMCHUN, Seoul, Korea) All other materials including Oil Red O powder, hematoxylin, sulfanilamide, N-ethylenediamine dihydrochloride, sodium nitrite, $( \pm)$ - $\alpha$-tocopherol (vitamin $\mathrm{E})$, catechin, quercitrin, and procyanidin B2 were purchased from Sigma-Aldrich (St. Louis, MO, USA). 


\subsection{Animals and Experimental Design}

A total of forty-eight C57BL/6N male mice (6-week old; 22-24 g) were purchased from Daehanbio-link (Eumseong-gun, Chung-book, Korea). The mice had free access to food pellets (Daehanbio-link) and water and were housed in a room maintained at $22 \pm 2{ }^{\circ} \mathrm{C}$ under a $12 \mathrm{~h}$ light: $12 \mathrm{~h}$ dark cycle. After acclimatization for 1 week, mice were divided randomly into six groups $(n=8)$ : the naive, control, EFAX (25, 50, and $100 \mathrm{mg} / \mathrm{kg})$, and vitamin E (100 mg/kg) groups. Mice were fed a $60 \%$ high fat and 20\% low carbohydrate diet (Research Diets Inc., New Brunswick, NJ, USA) for 8 weeks, excluding the naive group (normal chow diet, Supplementary Table S1). From the 3rd week of high fat diet feeding, distilled water (control), EFAX, and vitamin E were administered by gavage daily for the last 6 weeks. Food intake and body weight were measured twice a week. On the final day of the experiment, the mice were euthanized in $\mathrm{CO}_{2}$ Chamber (Jeungdo Bio\&Plant, Seoul, Korea) after a 6-h fast, and whole blood was collected from the abdominal aorta. The liver and adipose tissues (epididymal, retroperitoneal and visceral) were weighed and stored immediately.

The protocol was approved by the Institutional Animal Care and Use Committee of Daejeon University (DJUARB2020-008) and was conducted in accordance with the Guide for the Care and Use of Laboratory Animals, published by the National Institutes of Health (NIH, MD).

\subsection{Determination of Hepatic Lipid Levels and Serum Biochemistry}

Hepatic triglyceride (TG) and hepatic total cholesterol (TC) levels were determined using commercial kits (ASAN pharmacy, Seoul, Korea) according to a previous method [23]. Serum levels of aspartate aminotransferase (AST), alanine aminotransferase (ALT), TG, TC, low-density lipoprotein cholesterol (LDL), and high-density lipoprotein cholesterol (HDL) were determined using an autoanalyzer (Chiron, Emeryville, CA, USA).

\subsection{Histopathological Analysis in the Hepatic Tissue}

To evaluate hepatic fat accumulation, frozen liver samples were sectioned at $10 \mu \mathrm{m}$ and stained with Oil-Red O and Mayer's hematoxylin. For the histomorphological evaluation, the slides were stained with hematoxylin and eosin (H\&E). For immunohistochemical staining of myeloperoxidase (MPO) and 4-hydroxynonenal (4-HNE), the liver tissues were fixed in 10\% formalin, embedded in paraffin blocks, and cut into $5 \mu \mathrm{m}$ sections. Immunostaining was performed with anti-4-HNE antibody (Abcam, Cambridge, UK) or anti-MPO antibody (Abcam, Cambridge, UK), and a biotinylated secondary antibody (Vector Laboratories, Burlingame, CA, USA) was used. The slides were developed with $0.05 \%$ DAB (Sigma Aldrich, St. Louis, MO, USA) and then immediately counter-stained with Mayer's hematoxylin. Five photographs per sample were acquired using an optical microscope (Leica, Wetzlar, Germany) at 200× (for H\&E and 4-HNE) or 400× (for Oil-Red O and MPO) magnification.

\subsection{Determination of Inflammatory Cytokines in Hepatic Tissue}

Hepatic levels of inflammatory cytokines were measured using commercial ELISA kits: tumor necrosis factor (TNF)- $\alpha$ (BD Biosciences, San Jose, CA, USA) and interleukin (IL)- $1 \beta$ (R\&D Systems, Minneapolis, MN, USA).

\subsection{Determination of Nitric Oxide (NO) and Lipid Peroxidation in Hepatic Tissue}

The hepatic levels of NO were evaluated using the Griess method [24]. Hepatic levels of lipid peroxidation represented as malondialdehyde (MDA) were determined using thiobarbituric acid reactive substances (TBAR) according to a previous method [25]. The absorbance was measured at $540 \mathrm{~nm}$ for NO or 520-535 nm for MDA using a spectrophotometer (Molecular Device Corp., San Jose, CA, USA). 


\subsection{Western Blot Analysis for Lipid Metabolic Proteins in the Hepatic Tissue}

\subsubsection{Fatty Acid Metabolism-Related Proteins}

Western blot analysis evaluated five hepatic proteins related to fatty acid metabolism as follows: mature sterol regulatory element-binding protein (mSREBP)-1c, fatty acid synthase (FAS), peroxisome proliferator-activated receptor (PPAR)- $\alpha$, total AMP-activated protein kinase (AMPK)- $\alpha$, and phosphorylated-AMPK (pAMPK)- $\alpha$.

\subsubsection{TG Synthesis-Related Proteins}

Regarding TG synthesis, western blot analysis was conducted to evaluate three hepatic proteins as follows: glycerol-3-phosphate acyltransferase (GPAM), diglyceride acyltransferase (DGAT)1, and DGAT2.

\subsubsection{Western Blot Performance}

The proteins were separated by $10 \%$ polyacrylamide gel electrophoresis and transferred to polyvinylidene fluoride (PVDF) membranes. After blocking in 3\% BSA, the membranes were probed with primary antibodies (SREBP-1c, PPAR- $\alpha$, GPAM, DGAT1, and DGAT2 (Abcam, UK); FAS, AMPK- $\alpha$, and pAMPK- $\alpha$ (Cell Signaling Technology, Danvers, MA, CA, USA); $\beta$-actin from (Thermo Fisher Scientific, Waltham, MA, USA)) overnight at $4{ }^{\circ} \mathrm{C}$. The membranes were washed and incubated with an anti-rabbit IgG-HRP (GeneTex, Irvine, CA, USA) for $2 \mathrm{~h}$. Proteins were imaged using an advanced enhanced chemiluminescence (ECL) advanced kit (Thermo Fisher Scientific, CA, USA). Protein expression was observed using the FUSION Solo System (Vilber Lourmat, Marne-la-Vallée, France), and semi-quantified using ImageJ (NIH).

\subsection{Statistical Analysis}

The data are expressed as the means \pm standard deviation (SD) or fold changes in means. Statistical significance was determined by using one-way analysis of variance (ANOVA) followed by Dunnett's test. In all analyses, $p<0.05$ was taken to indicate statistical significance.

\section{Results}

\subsection{Food Intake and Body, Liver, and Adipose Tissue Weights}

As expected, the 8-week high-fat diet increased the average body weight to 1.5 -fold that of the naive group fed the normal diet, and the weights of liver and adipose tissues (epididymal, retroperitoneal, visceral, and total, $p<0.05$ or $p<0.01$ ) also increased. Administration of EFAX (especially $25 \mathrm{mg} / \mathrm{kg}$ and $100 \mathrm{mg} / \mathrm{kg}$ ) significantly attenuated these increases in all measurements: body weight $(p<0.05)$, absolute liver weight $(p<0.05$ or $p<0.01)$, and adipose tissue weight $(p<0.05)$, respectively (Figure 1A,B and Table 1). The positive control group receiving vitamin E $(100 \mathrm{mg} / \mathrm{kg}) \mathrm{did}$ not show any significant differences for those measurements.

In this experimental model, the consumed food weight differed between the naive (normal diet) and high-fat diet groups, but there was no significant difference between the control and treatment groups. Total calorie intake was not significantly different among all groups, including the naive group (Figure 1C,D). 
A
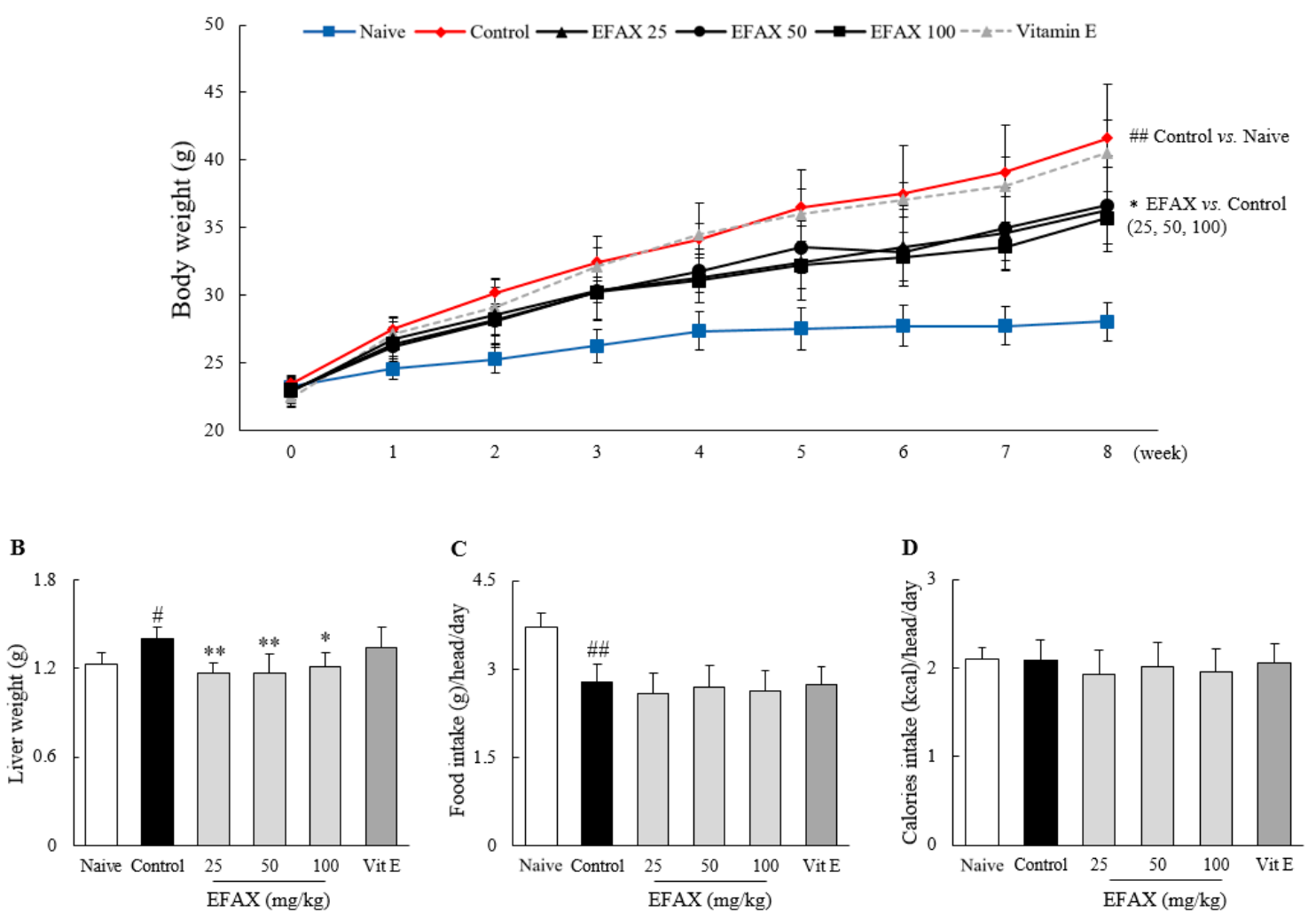

Figure 1. Effects on body weight, liver weight, and food (and calorie) intake. Mice ( $n=8$ in each group) were fed a $60 \%$ high-fat diet for 8 weeks, excluding the naive group (normal chow diet). From the 3rd week of high-fat diet feeding, distilled water (control), ethyl acetate fraction of Amomum xanthioides (EFAX) $(25 \mathrm{mg} / \mathrm{kg}, 50 \mathrm{mg} / \mathrm{kg}, 100 \mathrm{mg} / \mathrm{kg})$, and vitamin $\mathrm{E}(100 \mathrm{mg} / \mathrm{kg})$ were administered by gavage daily for the last 6 weeks. Body weight (A), liver weight (B), and food intake (C) were recorded, and then caloric intake (D) was calculated. ${ }^{\#} p<0.05,{ }^{\# \#} p<0.01$ compared with the naive group; ${ }^{*} p<0.05$, ** $p<0.01$ compared with the control group.

Table 1. Adipose tissue weights and serum lipid profiles.

\begin{tabular}{|c|c|c|c|c|c|c|c|}
\hline \multirow{2}{*}{\multicolumn{2}{|c|}{ Group }} & \multicolumn{6}{|c|}{ High-Fat Diet } \\
\hline & & Naive & Control & EFAX 25 & EFAX 50 & EFAX 100 & Vitamin E \\
\hline \multirow{4}{*}{$\begin{array}{l}\text { Adipose } \\
\text { tissue } \\
\text { weight }\end{array}$} & Epididymal (g) & $0.79 \pm 0.30$ & $3.16 \pm 0.13^{\# \#}$ & $2.45 \pm 0.40^{*}$ & $2.60 \pm 0.43$ & $2.47 \pm 0.35 *$ & $2.99 \pm 0.51$ \\
\hline & Retroperitoneal (g) & $0.26 \pm 0.10$ & $1.40 \pm 0.10^{\# \#}$ & $1.10 \pm 0.21$ & $1.12 \pm 0.23$ & $0.96 \pm 0.18 *$ & $1.31 \pm 0.33$ \\
\hline & Visceral (g) & $0.54 \pm 0.09$ & $1.20 \pm 0.10^{\# \#}$ & $0.85 \pm 0.17 *$ & $0.92 \pm 0.18$ & $0.85 \pm 0.12 *$ & $1.22 \pm 0.34$ \\
\hline & Total (g) & $1.60 \pm 0.40$ & $5.76 \pm 0.28 \#$ & $4.40 \pm 0.73 *$ & $4.64 \pm 0.82$ & $4.29 \pm 0.60 *$ & $5.52 \pm 1.15$ \\
\hline \multicolumn{2}{|c|}{ Total cholesterol (mg/dL) } & $89.9 \pm 9.1$ & $184.1 \pm 15.3^{\# \#}$ & $157.4 \pm 4.8 * *$ & $138.7 \pm 14.8^{* *}$ & $156.1 \pm 8.5^{* *}$ & $183.6 \pm 9.9$ \\
\hline \multicolumn{2}{|c|}{ LDL-C (mg/dL) } & $7.9 \pm 2.8$ & $22.1 \pm 1.6$ & $16.3 \pm 0.9^{* *}$ & $15.2 \pm 2.4^{* *}$ & $16.0 \pm 1.6^{* *}$ & $23.0 \pm 2.9$ \\
\hline \multicolumn{2}{|c|}{ HDL-C (mg/dL) } & $90.0 \pm 6.41$ & $126.4 \pm 8.7$ \#\# & $124.3 \pm 7.2$ & $118.5 \pm 9.5$ & $117.3 \pm 9.1$ & $126.6 \pm 8.2$ \\
\hline \multicolumn{2}{|c|}{ Triglyceride (mg/dL) } & $80.0 \pm 15.0$ & $44.2 \pm 14.9$ & $37.7 \pm 3.4$ & $36.2 \pm 6.3$ & $40.9 \pm 6.5$ & $45.3 \pm 11.4$ \\
\hline
\end{tabular}

\subsection{Effects on Hepatic Lipid Accumulation}

The high fat diet intake for 8 weeks remarkably induced the hepatic lipid accumulation, as evidenced by histopathologic findings using Oil Red O staining, with high levels of hepatic TG (4.6-fold) and TC (7.7-fold) compared to those in the naive group. EFAX treatments significantly ameliorated the deposition of hepatic lipid droplets $(p<0.01)$ and TG levels $(p<0.01)$ compared with those of the control. An effect of EFAX on hepatic TC levels was observed but did not show statistical 
significance. Vitamin E showed no effects on Oil Red O staining and hepatic TG levels but significantly reduced hepatic TC levels $(p<0.01$, Figure 2).

$\mathbf{A}$

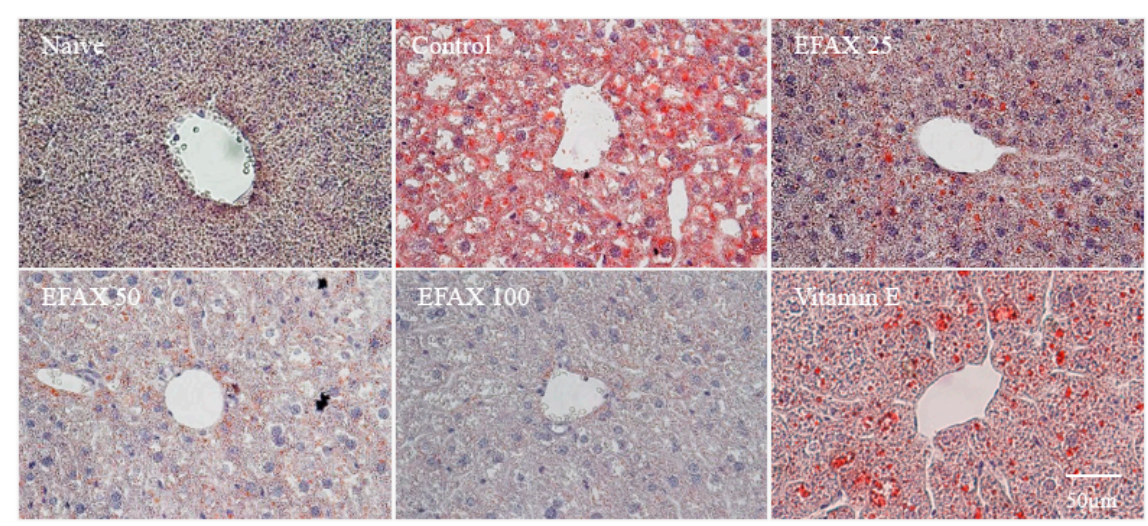

B

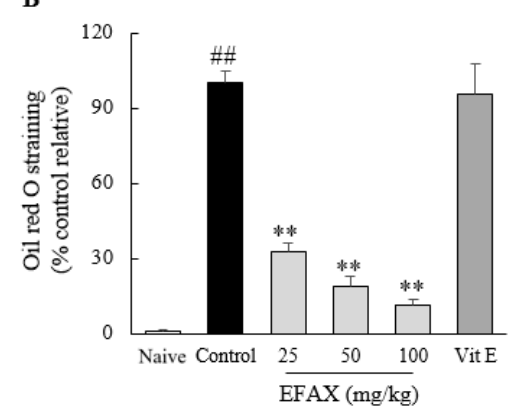

C

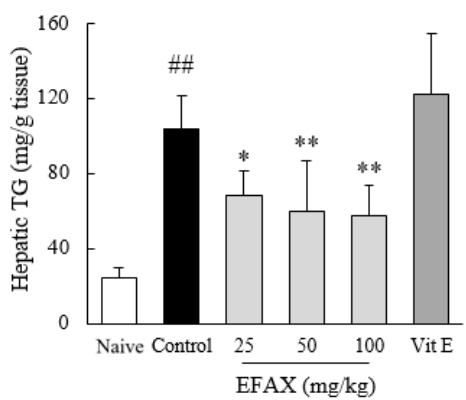

D

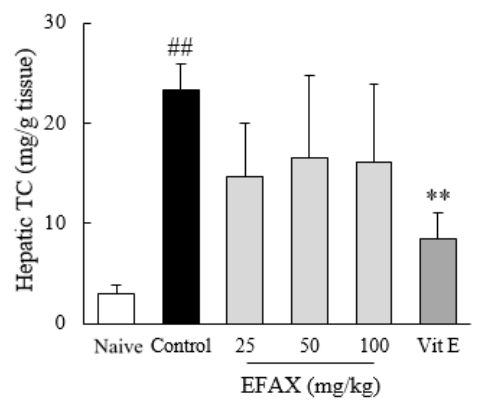

Figure 2. Effects on hepatic lipid profiles. Mice ( $n=8$ in each group) were fed a $60 \%$ high fat diet for 8 weeks excluding naive group (normal chow diet). From the 3rd week of high fat diet feeding, distilled water (control), EFAX ( $25 \mathrm{mg} / \mathrm{kg}, 50 \mathrm{mg} / \mathrm{kg}, 100 \mathrm{mg} / \mathrm{kg}$ ), and vitamin E (100 mg/kg) were administered by gavage daily for the last 6 weeks. Oil Red O staining (A) of liver sections was performed, and representative photographs (400× magnification) were semi-quantified (B). Hepatic levels of Hepatic triglyceride (TG) (C) and total cholesterol (TC) (D) were evaluated. \#\# $p<0.01$ compared with the naive group; ${ }^{*} p<0.05,{ }^{* *} p<0.01$ compared with the control group.

\subsection{Effects on Lipid Profiles in Serum}

The high-fat diet considerably elevated serum levels of TC (2.0-fold), LDL (2.8-fold), and HDL (1.4-fold) but reduced serum TG levels (0.6-fold). EFAX treatments significantly reduced these alterations in TC $(p<0.01)$ and LDL $(p<0.01)$ levels compared with those of the control, but no changes in either HDL or TG levels were observed. No significant effect on lipid profile was observed in the vitamin E-treated group (Table 1).

\subsection{Effects on Molecules for Fatty Acid-Metabolism}

The 8-week administration of a high-fat diet activated both "de novo lipogenesis"-related proteins (mSREBP-1c; 4.3-fold and FAS; 1.2-fold) and a "fatty acid $\beta$-oxidation"-related protein (PPAR- $\alpha$; 2.4-fold). EFAX treatments markedly reduced the alterations of hepatic SREBP-1c $(p<0.01$ for 50 and $100 \mathrm{mg} / \mathrm{kg})$ and FAS $(p<0.01)$ but significantly upregulated the PPAR- $\alpha$ levels $(p<0.01)$ compared with those of the control (Figure 3A-C). EFAX treatments also accelerated AMPK- $\alpha$, a molecule with a pro-lipolysis activity, evidenced by a marked elevation in the pAMPK/AMPK ratio $(p<0.01)$ compared with that of the control. Vitamin E also showed similar effects on mSREPB-1c $(p<0.05)$ and FAS 
$(p<0.01)$ as EFAX but reduced the activation of hepatic PPAR- $\alpha(p<0.01)$. Vitamin E had no effect on the pAMPK/AMPK ratio (Figure $3 \mathrm{~A}-\mathrm{C}$ ).


Figure 3. Effects on hepatic levels of lipid metabolic proteins. Mice ( $n=8$ in each group) were fed a $60 \%$ high-fat diet for 8 weeks, excluding the naive group (normal chow diet). From the 3rd week of high-fat diet feeding, distilled water (control), EFAX (25 mg/kg, $50 \mathrm{mg} / \mathrm{kg}, 100 \mathrm{mg} / \mathrm{kg}$ ), and vitamin $\mathrm{E}(100 \mathrm{mg} / \mathrm{kg})$ were administered by gavage daily for the last 6 weeks. Western blot analysis evaluated the hepatic level of eight-lipid metabolic proteins (A), and semi-quantifications were performed on sterol regulatory element-binding protein (mSREBP)-1c and fatty acid synthase (FAS) (B); peroxisome proliferator-activated receptor (PPAR)- $\alpha$, AMP-activated protein kinase (AMPK)- $\alpha$, and phosphorylated-AMPK (pAMPK)- $\alpha(\mathrm{C})$; and glycerol-3-phosphate acyltransferase (GPAM), diglyceride acyltransferase (DGAT)1, and DGAT2 (D). ${ }^{\# \#} p<0.01$ compared with the naive group; ${ }^{*} p<0.05,{ }^{* *} p<0.01$ compared with the control group.

\subsection{Effects on Molecules for TG Synthesis}

Administration of a high-fat diet significantly elevated the hepatic protein levels of GPAM (3.6-fold, playing a role in TG synthesis before secretion or storage) and DGAT2 (1.9-fold, TG storage) but reduced that of the DGAT1 protein (0.8-fold, TG secretion). These alterations were significantly ameliorated by EFAX treatments (especially at $50 \mathrm{mg} / \mathrm{kg}$ or $100 \mathrm{mg} / \mathrm{kg}, p<0.05$ or $p<0.01$ ) compared with the control. Vitamin E also showed significant effects on the hepatic levels of GPAM $(p<0.05)$ and DGAT2 $(p<0.01)$ but not on DGAT1 (Figure 3A,D).

\subsection{Effects on Hepatic Inflammation}

H\&E staining reveals an 8-week high-fat diet induced mild hepatic inflammation (the number of ballooned hepatocytes was increased but no hepatic fibrosis was observed), which was consistent with elevated serum levels of AST (1.5-fold) and ALT (3.2-fold) in the control (Figure 4). This was supported by the measurements of hepatic MPO (10.5-fold), TNF- $\alpha$ (1.9-fold), and IL-1 $\beta$ (1.3-fold), respectively (Figure 5). EFAX treatments considerably ameliorated these changes in H\&E staining; serum levels of AST $(p<0.05$ or $p<0.01)$ and ALT $(p<0.05$ or $p<0.01)$; and hepatic levels of MPO activities $(p<0.01)$, TNF- $\alpha(p<0.05$ or $p<0.01)$, and IL- $1 \beta(p<0.05$ or $p<0.01)$ compared with those of the control. No beneficial result, however, was observed in the vitamin E treatment group (Figures 4 and 5). 
A


Figure 4. Effects on hepatic injuries. Mice ( $n=8$ in each group) were fed a $60 \%$ high-fat diet for 8 weeks, excluding naive group (normal chow diet). From the 3rd week of high-fat diet feeding, distilled water (control), EF AX (25 mg/kg, $50 \mathrm{mg} / \mathrm{kg}, 100 \mathrm{mg} / \mathrm{kg})$, and vitamin E (100 mg/kg) were administered by gavage daily for the last 6 weeks. H\&E staining (A) of liver sections was performed, and representative photographs (200x magnification) were semi-quantified (B). The red arrows indicate ballooned hepatocytes. Serum levels of aspartate aminotransferase (AST) (C) and alanine aminotransferase (ALT) (D) were evaluated. ${ }^{\# \#} p<0.01$ compared with the naive group; * $p<0.05$, ** $p<0.01$ compared with the control group.
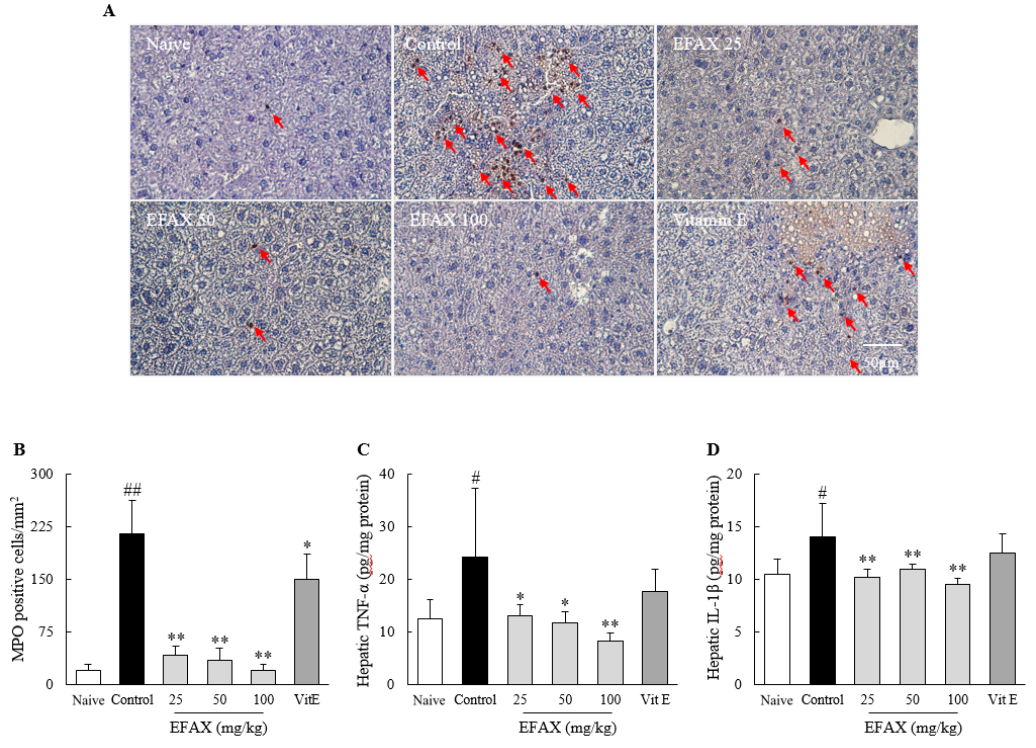

Figure 5. Effects on myeloperoxidase (MPO) staining and inflammatory cytokines. Mice ( $n=8$ in each group) were fed a $60 \%$ high-fat diet for 8 weeks, excluding the naive group (normal chow diet). From the 3rd week of high-fat diet feeding, distilled water (control), EFAX (25 mg/kg, $50 \mathrm{mg} / \mathrm{kg}$, $100 \mathrm{mg} / \mathrm{kg})$, and vitamin E $(100 \mathrm{mg} / \mathrm{kg})$ were administered by gavage daily for the last 6 weeks. MPO staining (A) of liver sections was performed, and representative photographs (400× magnification) were semi-quantified (B). Hepatic levels of tumor necrosis factor (TNF)- $\alpha$ (C) and interleukin (IL)-1 $\beta$ (D) were evaluated. ${ }^{\#} p<0.05,{ }^{\# \#} p<0.01$ compared with the naive group; ${ }^{*} p<0.05,{ }^{* *} p<0.01$ compared with the control group. 


\subsection{Effects on Hepatic Oxidative Stress}

As expected, an 8-week high-fat diet induced notable hepatic oxidative stress, evidenced by increases in hepatic levels of 4-HNE (12.7-fold), MDA (1.7-fold), and NO (2.1-fold). These alterations, namely, the levels of 4 -HNE $(p<0.01)$, MDA $(p<0.05)$, and NO $(p<0.01)$, were significantly attenuated by the EFAX treatments compared with those of the control. Vitamin E exerted considerable effects on hepatic 4-HNE $(p<0.01)$ and MDA $(p<0.01)$ levels but not on NO levels (Figure 6).
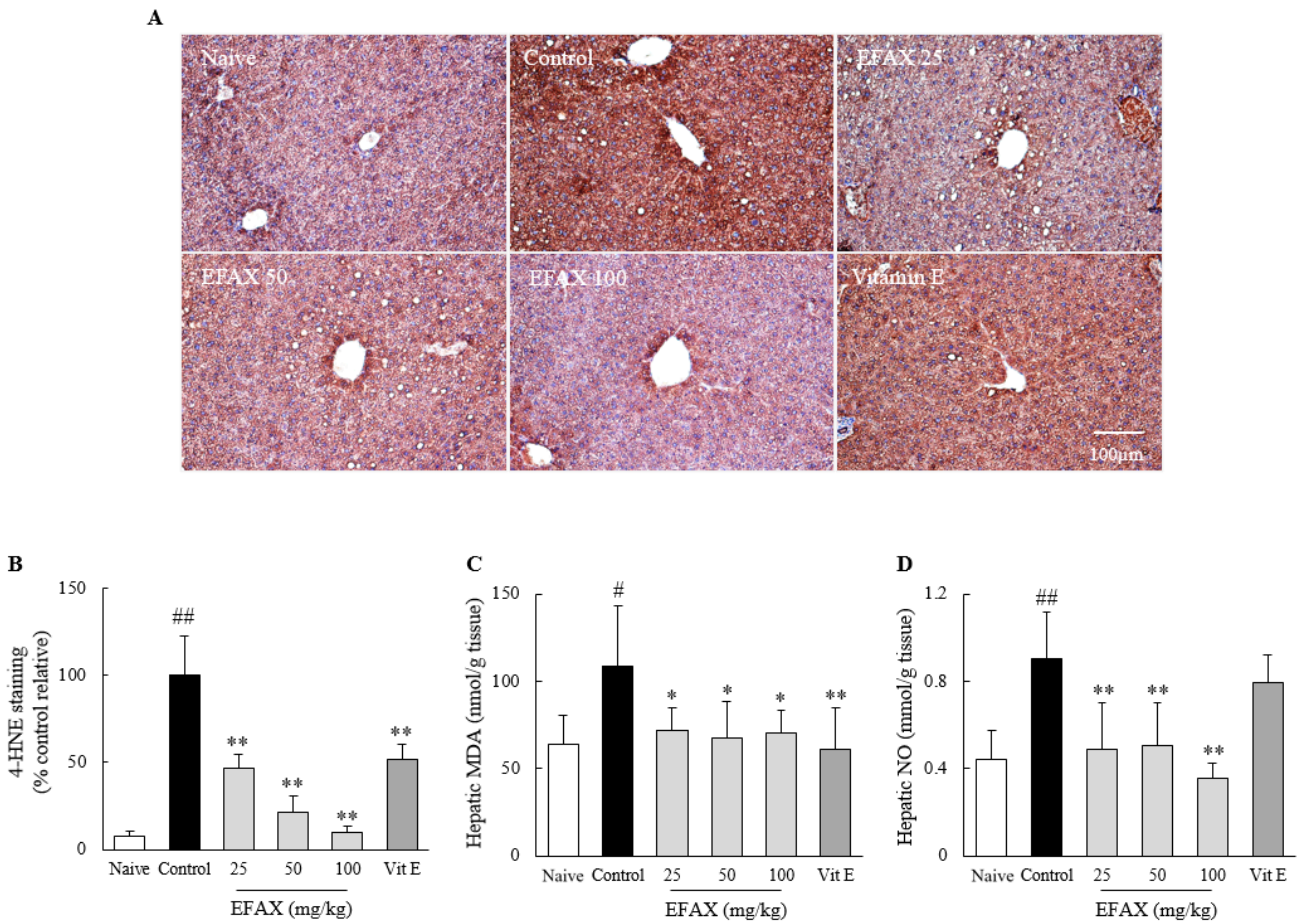

Figure 6. Effects on hepatic oxidative stress. Mice ( $n=8$ in each group) were fed a $60 \%$ high-fat diet for 8 weeks, excluding the naive group (normal chow diet). From the 3 rd week of high-fat diet feeding, distilled water (control), EFAX (25 mg/kg, $50 \mathrm{mg} / \mathrm{kg}, 100 \mathrm{mg} / \mathrm{kg})$, and vitamin E (100 mg/kg) were administered by gavage daily for the last 6 weeks. 4-hydroxynonenal (4-HNE) staining (A) of liver sections was performed, and representative photographs (200× magnification) were semi-quantified (B). Hepatic levels of malondialdehyde (MDA) (C) and Nitric Oxide (NO) (D) were evaluated. ${ }^{\#} p<0.05$, \#\# $p<0.01$ compared with the naive group; ${ }^{*} p<0.05,{ }^{* *} p<0.01$ compared with the control group.

\section{Discussion}

NAFLD is defined as hepatic disorders caused by fat accumulation ( $\geq 5 \%$ of the liver weight) in the absence of excessive alcohol consumption and its sequential diseases, including inflammation (NASH) and fibrotic changes (liver fibrosis and cirrhosis) [26,27]. The presence of NAFLD increases the risk of type 2 diabetes (1.6 5.5-fold) and mortality from cardiovascular diseases (approximately 2.0-fold) and chronic kidney diseases (1.4 1.9-fold) [4,28,29]. Clinical data reported that $20 \sim 80 \%$ of patients with NAFLD also have dyslipidemia [30]. As expected, the 8-week high-fat diet induced fatty liver and dyslipidemia in the present study. Then, EFAX treatments effectively protected the development of both fatty liver and the altered serum profiles of lipid contents except for TG (Figure 2). On the other hand, the present animal model significantly reduced serum TG levels, which can result from the lower amounts of carbohydrates (26.2\% vs. $72.0 \%)$ in the high-fat diet than in the normal diet (Table 1 , Supplementary Table S1). In fact, a number of clinical studies reported that a high-fat diet could lower serum TG levels [31-33].

NAFLD is a multisystem disease that involves dynamic interactions between the liver and various extrahepatic organs, including blood, adipose tissues, and muscles [34]. Obesity, excessive fat 
accumulation in adipose tissues, is an independent risk factor for NAFLD, and the global prevalence of obesity has increased up to 39.0\% [35]. Current lifestyles, such as sedentary behavior and overnutrition, are considered the main contributors to excess fat storage in body organs [36]. Accordingly, weight loss is the most powerful intervention for NAFLD, and can be obtained by diet therapy and/or exercise [7]. In this study, EFAX considerably ameliorated the increases in body weight, adipose tissue weight, and liver weight associated with a high-fat diet (Figure 1A,B, Table 1). These outcomes, including weight loss, did not result from a reduction in total dietary caloric intake (Figure 1D), which suggests the potential of EFAX as a therapeutic drug for NAFLD. Several nature-derived compounds, such as gallic acid and paeoniflorin, were previously reported to also exert reducing effects on body weight and adipose tissue weight, as well as on fatty liver, in a high-fat diet model [37,38]; however, berberine, a popular nature-derived agent used to treat NAFLD, did not affect body weight or liver weight [39].

The liver is a central organ for homeostasis of lipid metabolism through both lipogenesis and lipolysis as well as through import from or export to blood and secretion with bile acid [40]. To explore the mechanisms corresponding to the above results by EFAX, we analyzed molecules for lipid metabolism in the liver. In our data, EFAX exerted lipolysis-dominant activity with inhibition of de novo lipogenesis, as evidenced by modulations of PPAR $\alpha$ and AMPK versus mSREBP-1c and FAS (Figure 3). These molecules have been pharmaceutical targets, such as metformin targeting AMPK, a key modulator of fatty acid $\beta$-oxidation, for NAFLD [41]. In fact, AMPK activity (pAMPK/AMPK levels) was upregulated in our study, which would be the compensatory reaction against an overload of fat due to a high-fat diet, and this upregulation was further accelerated by EFAX treatments (Figure 3C). TG is the major lipid component in the liver of patients with NAFLD [42], and EFAX showed activity controlling hepatic lipid homeostasis (Figure 3). The molecules GPAM, DGAT2, and DGAT1 are well known to play important roles in the initiation of TG synthesis, storage of lipid droplets, and TG secretion into VLDL, respectively [43].

Broadly, NAFLD can be divided into simple steatosis (NAFL) and steatohepatitis (NASH), accounting for approximately $80 \sim 90 \%$ and $10 \sim 20 \%$ of all NAFLD cases [44]. NAFL itself is not medically problematic, but approximately $25 \%$ of NAFL develops into NASH and further progresses to fibrosis in 3 years, which are the critical issues in NAFLD [45]. This progression involves multiple contributors, such as oxidative stress and inflammation following the dysregulation of lipid metabolism [28]. The overload of free fatty acids in the liver can lead to inflammation through the induction of phagocytic activation and oxidative stress [46-48]. Although it is not certain whether inflammation is always preceded by fat accumulation, lipid-overload liver injury is still a dominant model explaining NASH pathogenesis [46]. As expected, EFAX exerted anti-inflammatory activities evidenced by hepatic enzymes of serum AST and ALT levels, along with inflammatory cytokines (TNF- $\alpha$, IL-1 $\beta$ ), MPO activity, and oxidative stress (4-HNE, MDA, NO), respectively (Figures 4-6). Prolonged inflammation in the liver could progress into hepatic fibrosis, cirrhosis, and sometimes hepatocarcinoma which determine liver disorder-related mortality [49]. Our previous studies presented anti-hepatofibrotic effects of EFAX using bile-duct ligation and DMN liver injury animal models [21,22]; however, this study first reports the therapeutic effects of EFAX against NAFLD.

In our research regarding drug development for NAFLD treatment, we evaluated the potential of EFAX from $A$. xanthioides, a traditional medicinal herb treating gastrointestinal, hepatic, and dyslipidemic disorders [20]. The main constituents of EFAX include procyanidin B2, catechin, and quercitrin (Supplementary Figure S2). Previous studies reported anti-fatty liver and anti-fibrotic effects of procyanidin B2 using animal models [50,51]. Several clinical studies revealed the beneficial effects of catechin-enriched green tea on NAFLD [52,53]. However, we still do not know the exact compounds accounting for the anti-NAFLD effects in EFAX, which we will investigate in the future. In the present study, we adapted vitamin E as a positive control. Vitamin E, a well-known anti-oxidant, has been clinically recommended as a plausible treatment for NAFLD [11]. In our experimental model, vitamin E showed notable effects on hepatic levels of TC and oxidative stress but no significant effects on hepatic levels of TG and inflammatory cytokines (TNF- $\alpha$ and IL-1 $\beta$ ). A number of previous 
clinical studies have suggested anti-NAFLD effects of vitamin E; however, these effects are still controversial [54-56]. We cannot assure the reasons for the non-positive effects of vitamin $E$ on hepatic TG levels in our study, but it was also controversial in other animal studies depending on study groups $[57,58]$. On the other hand, gut dysbiosis is attracting attention as a critical contributor to NAFLD [59], and a previous study revealed procyanidin B2 improve NAFLD via regulating gut microbiome [50]. Modulation of gut dysbiosis might be an underlying mechanism which could explain the anti-NAFLD effect of EFAX. Future study is needed to explore this possibility.

Taken together, the results of the present study revealed the therapeutic effects of EFAX on high-fat diet-induced NAFLD. The main mechanisms corresponding to these effects may involve the modulation of molecules related to hepatic lipolysis and lipogenesis as well as the secretion of lipids. Further studies are required to better understand the mechanisms and identifications of its active compounds.

Supplementary Materials: The following are available online at http://www.mdpi.com/2072-6643/12/8/2433/s1. Figure S1: Flow chart of the ethyl acetate fraction of Amomum xanthioides (EFAX) acquisition. Figure S2: Chemical constitutions and quantitative analysis of EFAX using ultrahigh-performance liquid chromatography-tandem mass spectrometry (UHPLC-MS/MS) chromatograms. Table S1: Composition of normal chow diet and 60\% high-fat diet.

Author Contributions: All six authors participated in drafting the manuscript. H.-J.I. and S.-J.H. wrote the manuscript and analyzed the data; J.-S.L., S.-B.L., and J.-Y.K. performed the experiments; and C.-G.S. supervised the entire process of the study. All authors have read and agreed to the published version of the manuscript.

Funding: This study was supported by a grant of the National Research Foundation of Korea (2019R1A2C2088201), Korea.

Conflicts of Interest: The authors declare no conflict of interest.

\section{References}

1. Younossi, Z.M.; Koenig, A.B.; Abdelatif, D.; Fazel, Y.; Henry, L.; Wymer, M. Global epidemiology of nonalcoholic fatty liver disease-Meta-analytic assessment of prevalence, incidence, and outcomes. Hepatology 2016, 64, 73-84. [CrossRef]

2. Younossi, Z.M.; Blissett, D.; Blissett, R.; Henry, L.; Stepanova, M.; Younossi, Y.; Racila, A.; Hunt, S.; Beckerman, R. The economic and clinical burden of nonalcoholic fatty liver disease in the United States and Europe. Hepatology 2016, 64, 1577-1586. [CrossRef] [PubMed]

3. Armstrong, M.J.; Adams, L.A.; Canbay, A.; Syn, W.-K. Extrahepatic complications of nonalcoholic fatty liver disease. Hepatology 2014, 59, 1174-1197. [CrossRef] [PubMed]

4. Musso, G.; Gambino, R.; Cassader, M.; Pagano, G. Meta-analysis: Natural history of non-alcoholic fatty liver disease (NAFLD) and diagnostic accuracy of non-invasive tests for liver disease severity. Ann. Med. 2011, 43, 617-649. [CrossRef] [PubMed]

5. Mantovani, A.; Zaza, G.; Byrne, C.D.; Lonardo, A.; Zoppini, G.; Bonora, E.; Targher, G. Nonalcoholic fatty liver disease increases risk of incident chronic kidney disease: A systematic review and meta-analysis. Metabolism 2018, 79, 64-76. [CrossRef]

6. Issa, D.; Patel, V.; Sanyal, A.J. Future therapy for non-alcoholic fatty liver disease. Liver Int. 2018, 38, 56-63. [CrossRef]

7. Romero-Gómez, M.; Zelber-Sagi, S.; Trenell, M. Treatment of NAFLD with diet, physical activity and exercise. J. Hepatol. 2017, 67, 829-846. [CrossRef]

8. Vilar-Gomez, E.; Martinez-Perez, Y.; Calzadilla-Bertot, L.; Torres-Gonzalez, A.; Gra-Oramas, B.; Gonzalez-Fabian, L.; Friedman, S.L.; Diago, M.; Romero-Gomez, M. Weight Loss Through Lifestyle Modification Significantly Reduces Features of Nonalcoholic Steatohepatitis. Gastroenterology 2015, 149, 367-378. [CrossRef]

9. Loomba, R.; Sanyal, A.J. The global NAFLD epidemic. Nat. Rev. Gastroenterol. Hepatol. 2013, 10, 686-690. [CrossRef]

10. Pydyn, N.; Miękus, K.; Jura, J.; Kotlinowski, J. New therapeutic strategies in nonalcoholic fatty liver disease: A focus on promising drugs for nonalcoholic steatohepatitis. Pharmacol. Rep. 2020, 72, 1-12. [CrossRef]

11. Chalasani, N.; Younossi, Z.; Lavine, J.E.; Diehl, A.M.; Brunt, E.M.; Cusi, K.; Charlton, M.; Sanyal, A.J. The diagnosis and management of non-alcoholic fatty liver disease: Practice Guideline by the American Association for the Study of Liver Diseases, American College of Gastroenterology, and the American Gastroenterological Association. Hepatology 2012, 55, 2005-2023. [CrossRef] [PubMed] 
12. EASL-EASD-EASO Clinical Practice Guidelines for the management of non-alcoholic fatty liver disease. J. Hepatol. 2016, 64, 1388-1402. [CrossRef] [PubMed]

13. Rinella, M.E. Nonalcoholic fatty liver disease: A systematic review. JAMA 2015, 313, 2263-2273. [CrossRef]

14. Miller, E.R.; Pastor-Barriuso, R.; Dalal, D.; Riemersma, R.A.; Appel, L.J.; Guallar, E. Meta-analysis: High-dosage vitamin E supplementation may increase all-cause mortality. Ann. Intern. Med. 2005, 142, 37-46. [CrossRef] [PubMed]

15. Lewis, J.D.; Ferrara, A.; Peng, T.; Hedderson, M.; Bilker, W.B.; Quesenberry, C.P.; Vaughn, D.J.; Nessel, L.; Selby, J.; Strom, B.L. Risk of Bladder Cancer Among Diabetic Patients Treated With Pioglitazone. Diabetes Care 2011, 34, 916-922. [CrossRef]

16. Colmers, I.N.; Bowker, S.L.; Majumdar, S.R.; Johnson, J.A. Use of thiazolidinediones and the risk of bladder cancer among people with type 2 diabetes: A meta-analysis. CMAJ Can. Med. Assoc. J. 2012, 184, E675-E683. [CrossRef]

17. Thiagarajan, P.; Aithal, G.P. Drug Development for Nonalcoholic Fatty Liver Disease: Landscape and Challenges. J. Clin. Exp. Hepatol. 2019, 9, 515-521. [CrossRef]

18. Yao, H.; Qiao, Y.-J.; Zhao, Y.-L.; Tao, X.-F.; Xu, L.-N.; Yin, L.-H.; Qi, Y.; Peng, J.-Y. Herbal medicines and nonalcoholic fatty liver disease. World J. Gastroenterol. 2016, 22, 6890-6905. [CrossRef]

19. Yan, T.; Yan, N.; Wang, P.; Xia, Y.; Hao, H.; Wang, G.; Gonzalez, F.J. Herbal drug discovery for the treatment of nonalcoholic fatty liver disease. Acta Pharm. Sin. B 2020, 10, 3-18. [CrossRef]

20. Suo, S.; Lai, Y.; Li, M.; Song, Q.; Cai, J.; Zhao, J.; Yang, Q.; Ung, C.O.L.; Hu, H. Phytochemicals, pharmacology, clinical application, patents, and products of Amomi fructus. Food Chem. Toxicol. 2018. [CrossRef]

21. Lee, S.-B.; Kim, H.-G.; Kim, H.-S.; Lee, J.-S.; Im, H.-J.; Kim, W.-Y.; Son, C.-G. Ethyl Acetate Fraction of Amomum xanthioides Exerts Antihepatofibrotic Actions via the Regulation of Fibrogenic Cytokines in a Dimethylnitrosamine-Induced Rat Model. Evid. Based Complement. Altern. Med. ECAM 2016, 2016. [CrossRef] [PubMed]

22. Kim, H.-G.; Han, J.-M.; Lee, J.-S.; Suk Lee, J.; Son, C.-G. Ethyl acetate fraction of Amomum xanthioides improves bile duct ligation-induced liver fibrosis of rat model via modulation of pro-fibrogenic cytokines. Sci. Rep. 2015, 5. [CrossRef] [PubMed]

23. Carr, T.P.; Andresen, C.J.; Rudel, L.L. Enzymatic determination of triglyceride, free cholesterol, and total cholesterol in tissue lipid extracts. Clin. Biochem. 1993, 26, 39-42. [CrossRef]

24. Tsikas, D. Analysis of nitrite and nitrate in biological fluids by assays based on the Griess reaction: Appraisal of the Griess reaction in the l-arginine/nitric oxide area of research. J. Chromatogr. B 2007, 851, 51-70. [CrossRef]

25. Kamal, A.-A.M.; Gomaa, A.; Khafif, M.E.; Hammad, A.S. Plasma lipid peroxides among workers exposed to silica or asbestos dusts. Environ. Res. 1989, 49, 173-180. [CrossRef]

26. Abd El-Kader, S.M.; El-Den Ashmawy, E.M.S. Non-alcoholic fatty liver disease: The diagnosis and management. World J. Hepatol. 2015, 7, 846-858. [CrossRef]

27. Angulo, P. Nonalcoholic Fatty Liver Disease. N. Engl. J. Med. 2002, 346, 1221-1231. [CrossRef]

28. Byrne, C.D.; Targher, G. NAFLD: A multisystem disease. J. Hepatol. 2015, 62, S47-S64. [CrossRef]

29. Musso, G.; Gambino, R.; Tabibian, J.H.; Ekstedt, M.; Kechagias, S.; Hamaguchi, M.; Hultcrantz, R.; Hagström, H.; Yoon, S.K.; Charatcharoenwitthaya, P.; et al. Association of non-alcoholic fatty liver disease with chronic kidney disease: A systematic review and meta-analysis. PLoS Med. 2014, 11, e1001680. [CrossRef]

30. de Araújo Souza, M.R.; de Melo Diniz, M.d.F.F.; de Medeiros-Filho, J.E.M.; de Araújo, M.S.T. Metabolic syndrome and risk factors for non-alcoholic fatty liver disease. Arq. Gastroenterol. 2012, 49, 89-96. [CrossRef]

31. Jacobs, B.; De Angelis-Schierbaum, G.; Egert, S.; Assmann, G.; Kratz, M. Individual Serum Triglyceride Responses to High-Fat and Low-Fat Diets Differ in Men with Modest and Severe Hypertriglyceridemia. J. Nutr. 2004, 134, 1400-1405. [CrossRef] [PubMed]

32. Pieke, B.; von Eckardstein, A.; Gülbahçe, E.; Chirazi, A.; Schulte, H.; Assmann, G.; Wahrburg, U. Treatment of hypertriglyceridemia by two diets rich either in unsaturated fatty acids or in carbohydrates: Effects on lipoprotein subclasses, lipolytic enzymes, lipid transfer proteins, insulin and leptin. Int. J. Obes. Relat. Metab. Disord. J. Int. Assoc. Study Obes. 2000, 24, 1286-1296. [CrossRef] [PubMed] 
33. West, C.E.; Sullivan, D.R.; Katan, M.B.; Halferkamps, I.L.; van der Torre, H.W. Boys from populations with high-carbohydrate intake have higher fasting triglyceride levels than boys from populations with high-fat intake. Am. J. Epidemiol. 1990, 131, 271-282. [CrossRef] [PubMed]

34. Reccia, I.; Kumar, J.; Akladios, C.; Virdis, F.; Pai, M.; Habib, N.; Spalding, D. Non-alcoholic fatty liver disease: A sign of systemic disease. Metabolism 2017, 72, 94-108. [CrossRef]

35. Chooi, Y.C.; Ding, C.; Magkos, F. The epidemiology of obesity. Metabolism 2019, 92, 6-10. [CrossRef]

36. Harrison, S.A.; Day, C.P. Benefits of lifestyle modification in NAFLD. Gut 2007, 56, 1760-1769. [CrossRef]

37. Chao, J.; Huo, T.-I.; Cheng, H.-Y.; Tsai, J.-C.; Liao, J.-W.; Lee, M.-S.; Qin, X.-M.; Hsieh, M.-T.; Pao, L.-H.; Peng, W.-H. Gallic acid ameliorated impaired glucose and lipid homeostasis in high fat diet-induced NAFLD mice. PLoS ONE 2014, 9, e96969. [CrossRef]

38. Zhang, L.; Yang, B.; Yu, B. Paeoniflorin Protects against Nonalcoholic Fatty Liver Disease Induced by a High-Fat Diet in Mice. Biol. Pharm. Bull. 2015, 38, 1005-1011. [CrossRef]

39. Guo, T.; Woo, S.-L.; Guo, X.; Li, H.; Zheng, J.; Botchlett, R.; Liu, M.; Pei, Y.; Xu, H.; Cai, Y.; et al. Berberine Ameliorates Hepatic Steatosis and Suppresses Liver and Adipose Tissue Inflammation in Mice with Diet-induced Obesity. Sci. Rep. 2016, 6. [CrossRef]

40. Musso, G.; Gambino, R.; Cassader, M. Recent insights into hepatic lipid metabolism in non-alcoholic fatty liver disease (NAFLD). Prog. Lipid Res. 2009, 48, 1-26. [CrossRef]

41. Smith, B.K.; Marcinko, K.; Desjardins, E.M.; Lally, J.S.; Ford, R.J.; Steinberg, G.R. Treatment of nonalcoholic fatty liver disease: Role of AMPK. Am. J. Physiol. Endocrinol. Metab. 2016, 311, E730-E740. [CrossRef] [PubMed]

42. Kawano, Y.; Cohen, D.E. Mechanisms of hepatic triglyceride accumulation in non-alcoholic fatty liver disease. J. Gastroenterol. 2013, 48, 434-441. [CrossRef] [PubMed]

43. Alves-Bezerra, M.; Cohen, D.E. Triglyceride Metabolism in the Liver. In Comprehensive Physiology; Terjung, R., Ed.; John Wiley \& Sons, Inc.: Hoboken, NJ, USA, 2017; pp. 1-22, ISBN 978-0-470-65071-4.

44. Hashimoto, E.; Taniai, M.; Tokushige, K. Characteristics and diagnosis of NAFLD/NASH. J. Gastroenterol. Hepatol. 2013, 28, 64-70. [CrossRef]

45. Fan, J.-G.; Kim, S.-U.; Wong, V.W.-S. New trends on obesity and NAFLD in Asia. J. Hepatol. 2017, 67, $862-873$. [CrossRef] [PubMed]

46. Friedman, S.L.; Neuschwander-Tetri, B.A.; Rinella, M.; Sanyal, A.J. Mechanisms of NAFLD development and therapeutic strategies. Nat. Med. 2018, 24, 908-922. [CrossRef] [PubMed]

47. Csak, T.; Ganz, M.; Pespisa, J.; Kodys, K.; Dolganiuc, A.; Szabo, G. Fatty acid and endotoxin activate inflammasomes in mouse hepatocytes that release danger signals to stimulate immune cells. Hepatology 2011, 54, 133-144. [CrossRef] [PubMed]

48. Klebanoff, S.J. Myeloperoxidase: Friend and foe. J. Leukoc. Biol. 2005, 77, 598-625. [CrossRef] [PubMed]

49. Ekstedt, M.; Franzén, L.E.; Mathiesen, U.L.; Thorelius, L.; Holmqvist, M.; Bodemar, G.; Kechagias, S. Long-term follow-up of patients with NAFLD and elevated liver enzymes. Hepatology 2006, 44, 865-873. [CrossRef]

50. Xing, Y.-W.; Lei, G.-T.; Wu, Q.-H.; Jiang, Y.; Huang, M.-X. Procyanidin B2 protects against diet-induced obesity and non-alcoholic fatty liver disease via the modulation of the gut microbiota in rabbits. World J. Gastroenterol. 2019, 25, 955-966. [CrossRef]

51. Feng, J.; Wang, C.; Liu, T.; Li, J.; Wu, L.; Yu, Q.; Li, S.; Zhou, Y.; Zhang, J.; Chen, J.; et al. Procyanidin B2 inhibits the activation of hepatic stellate cells and angiogenesis via the Hedgehog pathway during liver fibrosis. J. Cell. Mol. Med. 2019, 23, 6479-6493. [CrossRef]

52. Mahmoodi, M.; Hosseini, R.; Kazemi, A.; Ofori-Asenso, R.; Mazidi, M.; Mazloomi, S.M. Effects of green tea or green tea catechin on liver enzymes in healthy individuals and people with nonalcoholic fatty liver disease: A systematic review and meta-analysis of randomized clinical trials. Phytother. Res. 2020. [CrossRef] [PubMed]

53. Sakata, R.; Nakamura, T.; Torimura, T.; Ueno, T.; Sata, M. Green tea with high-density catechins improves liver function and fat infiltration in non-alcoholic fatty liver disease (NAFLD) patients: A double-blind placebo-controlled study. Int. J. Mol. Med. 2013, 32, 989-994. [CrossRef] [PubMed]

54. Caldwell, S. NASH Therapy: Omega 3 supplementation, vitamin E, insulin sensitizers and statin drugs. Clin. Mol. Hepatol. 2017, 23, 103-108. [CrossRef] 
55. Lavine, J.E.; Schwimmer, J.B.; Molleston, J.P.; Murray, K.F.; Rosenthal, P.; Abrams, S.H.; Scheimann, A.O.; Sanyal, A.J.; Chalasani, N.; Tonascia, J.; et al. Effect of Vitamin E or Metformin for Treatment of Nonalcoholic Fatty Liver Disease in Children and Adolescents. JAMA 2011, 305, 1659-1668. [CrossRef] [PubMed]

56. Hadi, H.E.; Vettor, R.; Rossato, M. Vitamin E as a Treatment for Nonalcoholic Fatty Liver Disease: Reality or Myth? Antioxidants 2018, 7, 12. [CrossRef]

57. Sakr, H.F.; Abbas, A.M.; Haidara, M.A. Swimming, but not vitamin E, ameliorates prothrombotic state and hypofibrinolysis in a rat model of nonalcoholic fatty liver disease. J. Basic Clin. Physiol. Pharmacol. 2018, 29, 61-71. [CrossRef]

58. Şekeroğlu, V.; Aydın, B.; Atlı Şekeroğlu, Z.; Özdener Kömpe, Y. Hepatoprotective effects of capsaicin and alpha-tocopherol on mitochondrial function in mice fed a high-fat diet. Biomed. Pharmacother. 2018, 98, 821-825. [CrossRef]

59. Leung, C.; Rivera, L.; Furness, J.B.; Angus, P.W. The role of the gut microbiota in NAFLD. Nat. Rev. Gastroenterol. Hepatol. 2016, 13, 412-425. [CrossRef]

(C) 2020 by the authors. Licensee MDPI, Basel, Switzerland. This article is an open access article distributed under the terms and conditions of the Creative Commons Attribution (CC BY) license (http://creativecommons.org/licenses/by/4.0/). 